\title{
Performance and Wake Characteristics of a Tidal Turbine under Yaw
}

\author{
Pranav K. Modali, Nitin S. Kolekar, and Arindam Banerjee ${ }^{\#}$ \\ \# Department of Mechanical Engineering \& Mechanics, Lehigh University \\ 19 Memorial Drive West, Bethlehem, PA 18015 USA \\ Email for correspondence: arb612@lehigh.edu
}

\begin{abstract}
In tidal streams and rivers, the flow of water can be at yaw to the turbine rotor plane causing performance degradation and a skewed downstream wake. The current study aims to quantify the performance variation and associated wake behavior caused by a tidal turbine operating in a yawed inflow environment. A three-dimensional computational fluid dynamics study was carried out using multiple reference frame approach using $\kappa-\omega S S T$ turbulence model with curvature correction. The computations were validated by comparison with experimental results on a 1:20 scale prototype for $\mathrm{a}^{\circ}$ yaw case performed in a laboratory flume. The simulations were performed using a threebladed, constant chord, untwisted tidal turbine operating at uniform inflow. Yaw effects were observed for angles ranging from $5^{\circ}$ to $15^{\circ}$. An increase in yaw over this range caused a power coefficient deficit of $26 \%$ and a thrust coefficient deficit of about $8 \%$ at a tip speed ratio of 5 that corresponds to the maximum power coefficient for the tested turbine. In addition, wake propagation was studied up to a downstream distance of ten rotor radius, and skewness in the wake, proportional to yaw angle was observed. At higher yaw angles, the flow around the turbine rotor was found to cushion the tip vortices, accele rating the interaction between the tip vortices and the skewed wake, the reby facilitating a faster wake recovery. The center of the wake was tracked using a center of mass technique. The center of wake analysis was used to better quantify the deviation of the wake with increasing yaw angle. It was observed that with an increase in yaw angle, the recovery distance moved closer to the rotor plane. The wake was noticed to meander around the turbine centerline with increasing downstream distance and slightly deviate towards the free surface above the turbine centerline, magnitude of which varied depending on yaw.
\end{abstract}

Keywords - tidal turbine, yaw, wake propagation, wake analysis

\section{INTRODUCTION}

It was estimated that entire tidal energy resource in the US have the potential to generate approximately $65.87 \mathrm{GW}$ a year, which was roughly $15 \%$ of the annual electricity generation in the United States (2016)[1]. Historically, the European Union has spearheaded development and deployments of hydrokinetic energy converters, providing for $50 \%$ of tidal energy and $45 \%$ of wave energy $R \& D$ investments [2]. Several device designs have been explored over the last three decades, and a horizontal axis turbine has emerged as one of the popular designs for tidal current applications with several leading developers using this concept in their device design. The amount of energy extracted by a horizontal axis tidal turbine was directly proportional to the area swept by the turbine. From a practical and cost-effective point of view, the swept area can be maximized by placing multiple turbines in an array configuration with optimum diameter rather than single large diameter turbine [3], a practice commonly adopted for wind farms. In such scenarios, the wake generated by the upstream turbines may cause significant disturbance to the downstream turbines; a scenario that is enhanced if the incoming flow was at an angle (commonly referred to as yaw) to the turbine axis due to changes in the direction of tides (ebb and flow tides) or wave-current interaction [4] that occur at the mouths of tidal estuaries. Gooch et al., [5] using a power law approximation, demonstrated that there was $70 \%$ more extractable power (in the form of density, water velocity) on the upper half of the tidal stream than the lower half, making it the optimum location for turbine placement [6, 7]. A study by European Marine Energy Centre's (EMEC) [8] for a tidal channel of total depth of $45 \mathrm{~m}$ reported that the effect of surface waves can be experienced up to a depth of up to $44 \%$ from the freesurface, whereas the turbulence from bottom boundary layer propagates to around $37 \%$ from the bottom surface [8,9], exposing the top half of the stream to elevated levels of turbulence and yaw/pitch effects. Quantification of turbine performance and downstream wake on the variation of yaw angle was thus essential for design layout of a tidal farm for maximizing its power output.

Krogstad and Adaramola [10], studied the near wake effects and performance change in the wind turbine due to yaw. They observed a gradual deviation in the wake with an increasing non-uniformity as the yaw angle increases. Adaramola and Krogstad [11] observed that the cumulative power output from a wind farm could be increased by adjusting the tip speed ratio [henceforth referred to as $T S R$ ] for a yawed upstream turbine, to increase the power output of the downstream turbine placed at an optimum distance. It was observed that when the upstream turbine was operated at appropriate yaw and the downstream turbine was placed at a small distance; the overall efficiency was better when compared to having a larger distance between turbines and upstream turbine operating at zero yaw angles [11]. This was attributed to the increase in the power output of a downstream turbine due to the increased thrust experienced by it due to the yawed operating condition of the upstream turbine $[11,12]$. The power in the downstream turbines was noticed to increase by $29 \%$ for yaw of $40^{\circ}$ to the upstream turbine, relative to $4 \%$ increase in the power for upstream turbine yaw of $10^{\circ}$. Loland 
[13] studied wake propagation of wind turbines under different yaw conditions. The wake recovery time was reported to be inversely proportional to yaw angle and was attributed to the increased momentum interaction between the free-stream flow and the wake flow. Cleijne [14] measured the wake turbulence characteristics in the Sexbierum wind farm (in the Netherlands) and noticed the shear stress behavior to be similar to velocity shear. The turbulence in the core of wake was noticed to be more isotropic than the free-stream, although, some local peaks in turbulence intensity in the direction of the wind was observed, similar to results reported by Smith [15]. Medici [16] studied the wake propagation and vortex shedding for wind turbines at yaw and observed that recovery of velocity deficit was faster for increasing yaw angle due to high-energy mixing and shorter life of tip vortices. Howland et al.[17] conducted an experimental study on a non-rotating wind turbine in yaw to study realistic wake deflection. The center of the wake was calculated by Trujillo et al. [18] as the center of mass of velocity deficit at locations downstream from the turbine. Howland's study observed the formation of a skewed wake, which was consistent as it traveled downstream. A velocity deficit shift in the wake was noticed, predominantly near the wake center compared to top and bottom, which experience the shift in opposite direction. This was attributed to a set of counter-rotating vortices created due to the yaw of the turbine which deformed the wake. This turning around results in the wake to miss the downstream turbine completely as it wraps around the downstream rotor [17]. In summary, these studies have helped to better understand the cumulative performance of wind turbines and the evolution of the downstream wake due to varied inflow conditions.

Very few studies have been reported to understand the effect of yaw on the performance and wake generated by a tidal turbine. Earlier experimental studies focused on using a scaled commercial turbine in yawed flows to study turbine performance [19, 20]. Galloway [6, 7] used a combination of Blade Element Momentum (BEM) theory and laboratory experiments to study the effects of inflow misalignment a 3 bladed horizontal axis tidal stream turbine. Yaw Angles of $0^{\circ}$, $7.5^{\circ}, 15^{\circ}$ and $22.5^{\circ}$ in inflow due to wave-current interaction was investigated. It was observed that the turbine rotor captures less power and rotor thrust [21], resulting in reduced performance as the yaw angle increased. Galloway also observed that the wake skew angle generated was always slightly greater than the yaw angle of the upstream flow due to flow acceleration around the turbine [9]. Galloway's study primarily focused on cyclic loading and accelerated fatigue effects on rotor due to wave and misaligned flows on tidal stream turbine. The focus of the study was on dynamic loads experienced by a turbine due to yawed inflow and the authors stopped short of discussing the meandering of the downstream wake; a phenomenon of critical importance when designing farm layouts as reported in wind turbine literature. Tian et al.[22, 23] performed three-dimensional transient CFD calculations to study the effect of yaw angle and turbulence intensity on the performance of an in-stream $20 \mathrm{KW}$ hydrokinetic turbine. They report a drop in power and thrust coefficients as the yaw angle increased. Two-dimensional velocity contours of the downstream wake structure were presented, and it was concluded that the wake expansion rate was similar for all yaw cases. Park et al. [24] performed a fluid-structure interaction analysis to study the influence of yaw on performance and blade deformation. Frost et al. [25] conducted a CFD study to understand the performance variation due to a support structure upstream or downstream of the turbine; the proximity between the support stanchion and the rotor was varied. A reduction in performance was noted when the turbine was placed in the shadow region of its support stanchion. The authors opined that a yaw mechanism would be superior from a performance perspective in comparison to a bi-directional system; similar observations were made by Adaramola and Krogstad [11] for a wind turbine. We present a three-dimensional steady-state CFD study to characterize wake propagation and recovery at different downstream locations from the rotor plane that was subjected to a yawed inflow. Wake meandering was characterized based on tracking the angle of skew of wake centerline about the principal direction of flow.

\section{NUMERICAL METHODOLOGY}

\section{A. Domain and Turbine Model Description}

The model consists of a three-bladed horizontal axis turbine, with a radius $(R)$ of $0.1397 \mathrm{~m}$ that used an SG-6043 hydrofoil. The dimensions were chosen to mimic a 1:20 scale model turbine that we have used in previous studies[26]. The schematic of the domain used for the computational study can be seen in Figure 1. We denote the yaw angle as $\gamma$ and the skew angle for the wake centerline as $\alpha$. The cross-sectional area of the computational domain was $1.2192 \mathrm{~m} \times 1.2192 \mathrm{~m}$ and was based on an independent study conducted by the authors to eliminate any blockage effects; thereby avoiding using any blockage correction methods. Domain sizes of $0.6096 \mathrm{~m} \times 0.6096 \mathrm{~m}$ (that mimics the size of our water tunnel test section), $1.2192 \mathrm{~m} \times 1.2192 \mathrm{~m}$ and $2.4384 \mathrm{~m} \times 2.4384 \mathrm{~m}$ with the corresponding area based blockage ratios of $16.5 \%$, $4.125 \%$, and $1 \%$ respectively for our model turbine were simulated. The chosen domain size was selected to keep the blockage ratio below $5 \%$. The domain consists of inner

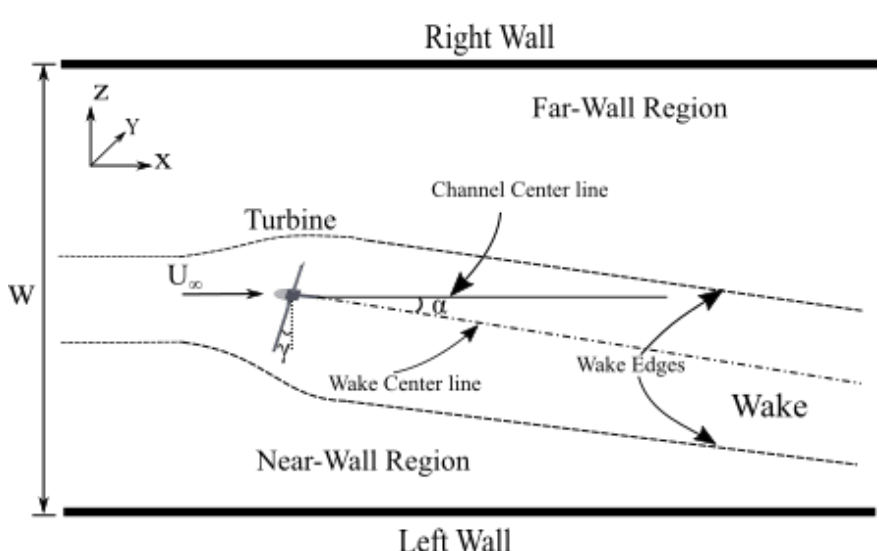

Figure 1: Flow schematic around tidal turbine 


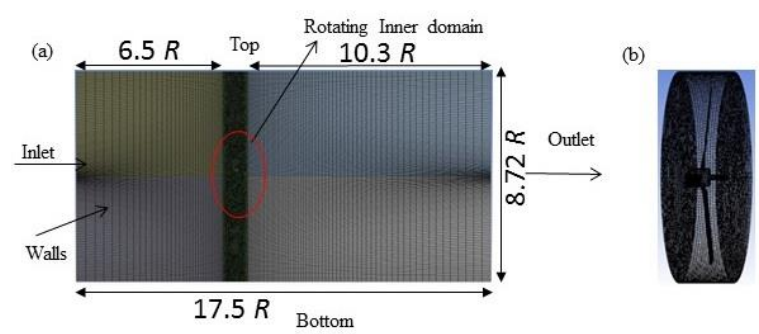

Figure 2: (a) Mesh used for CFD (19 million elements) (b) Inner fluid domain (1 million elements)

rotating sub-domain in which turbine was located and a stationary outer sub-domain. The size of the internal subdomain was $2.07 R \times 0.55 R$. The turbine was located at a distance of $6.5 R$ from the inlet, $10 R$ from the outlet and $4 R$ from the free surface. The mesh was optimized based on $y^{+}$ values $\left(y^{+}<10\right.$ was acceptable for proper prediction of boundary layer separation [26-28]) on the turbine. A Reynolds averaged Navier-Stokes (RANS) equations using the $\kappa-\omega$ SST (shear stress transport) turbulence model with curvature correction (CC) was solved. Torque based grid convergence study was performed by varying mesh size from 11 million to 26 million elements; an optimum size of 19 million elements was chosen to reduce computational costs. The torque obtained for 19 million elements had less than $3 \%$ variation when compared to the finest mesh size of 26 million elements. The mesh used for our current study is shown in Figure 2. Our previous CFD simulations [26, 29] on the same model turbine demonstrated Reynolds number (based on turbine diameter) converges $2.04 \times 10^{5}$; a uniform inlet flow speed of $0.73 \mathrm{~m} / \mathrm{s}$ was thus chosen for analysis and cross-comparison with previous work [26]. The channel outlet was specified as an outlet boundary condition with the average relative pressure of zero. The turbine and side-walls of the channel were modeled as no-slip walls, and the channel top was modeled as zero relative opening pressure. Multiple reference frame approach was used for steady-state simulations. The simulation was set to converge after the scaled root mean square residuals of continuity, momentum and turbulence quantities falls below $1 \times 10^{-5}$. Details of simulation variables are listed below in Table 1 .

Table 1. Parameters for CFD Analysis

\begin{tabular}{|c|c|}
\hline Hydrofoil Profile & SG -6043 \\
\hline Density $(\rho)$ & $998.2 \mathrm{~kg} / \mathrm{m}^{3}$ \\
\hline Pressure $(\mathrm{p})$ & $101.3 \mathrm{kPa}$ \\
\hline Rotor radius $(\mathrm{R})$ & $0.1397 \mathrm{~m}$ \\
\hline Chord length $(\mathrm{c})$ & $0.0165 \mathrm{~m}$ \\
\hline Number of Blades & 3 \\
\hline Rotor Speed $(\mathrm{rpm})$ & $50-350$ \\
\hline Freestream Velocity $\left(U_{\infty}\right)$ & $0.73 \mathrm{~m} / \mathrm{s}$ \\
\hline $\begin{array}{c}\text { CFD Convergence } \\
\text { criteria }\end{array}$ & $1 \times 10^{-5}$ \\
\hline $\begin{array}{c}\text { Reynolds Number } \\
\text { (Diameter based) }\end{array}$ & $2.04 \times 10^{5}$ \\
\hline
\end{tabular}

B. Governing Equations \& Non-dimensional Parameters
Non-dimensional parameters that govern the performance of the turbine were $T S R(\lambda)$, the power coefficient $\left(C_{p}\right)$, and coefficient of thrust $\left(C_{T}\right)$ which were defined as:

$$
\begin{gathered}
\lambda=R \Omega / U_{\infty} \\
C_{p}=\frac{P_{\text {out }}}{0.5 \rho A U_{\infty}^{3}} \\
C_{T}=\frac{T}{0.5 \rho A U_{\infty}^{2}}
\end{gathered}
$$

where $R$ was the radius, $\Omega$ rotor rotational speed of the turbine in radians/second, $U_{\infty}$ was the freestream velocity, $P_{\text {out }}$ was power output of the turbine; $\rho$ was the density of the fluid, $A$ was the swept area of the turbine blades, and $T$ was the thrust force on the rotor plane.

For the present case, three-dimensional unsteady Reynolds-averaged Navier-stokes equations with $\kappa-\omega$ SST turbulence model with curvature correction (CC) was solved [30, 31]. A multiple reference frame technique is adopted similar to our previous studies [26, 29]; a rotation frame (in our case, the inner fluid domain) takes into account the effect of turbine rotation by transforming an unsteady flow in inertial frame (stationary) to a steady flow in non-inertial frame (rotating). The mass and momentum conservation equations can be written as

$$
\begin{aligned}
& \nabla \cdot \stackrel{1}{U}_{r}=0
\end{aligned}
$$

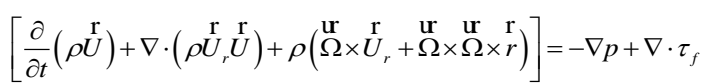

where $\vec{U}_{r}=(=\vec{U}-\vec{\Omega} \times \vec{r})$ is the relative velocity viewed from a rotating reference frame, $\rho\left(\vec{\Omega} \times \vec{U}_{r}\right)$ is Coriolis force, $\rho(\vec{\Omega} \times \vec{\Omega} \times \vec{r})$ is the centrifugal force, $\nabla p$ is the pressure gradient across the turbine and $\tau_{f}$ is viscous stress tensor.

The two equation $\kappa-\omega$ SST eddy viscosity model has been used for its efficiency to predict complex fluid flows under a broad range of adverse pressure gradient flow conditions $[31,32]$. The production term in both the $k$ and $\omega$ equations was scaled with a curvature correction term $f_{\mathrm{r} 1}$ as:

$$
\begin{gathered}
\frac{\partial(\rho k)}{\partial t}+\frac{\partial\left(\rho u_{j} k\right)}{\partial x_{j}}=P_{k} f_{r 1}-\beta^{*} \rho k \omega+\frac{\partial}{\partial x_{j}}\left[\mu_{e f f} \frac{\partial k}{\partial x_{j}}\right] \\
\frac{\partial(\rho \omega)}{\partial t}+\frac{\partial\left(\rho u_{j} \omega\right)}{\partial x_{j}}=\alpha \rho \frac{P_{k}}{\mu_{t}} f_{r 1}-D_{\omega}+C d_{\omega}+\frac{\partial}{\partial x_{j}}\left[\mu_{e f f} \frac{\partial \omega}{\partial x_{j}}\right]
\end{gathered}
$$

where the modified function is defined as:

$$
f_{r 1}=\max \left\{\min \left(f_{\text {rotation }}, 1.25\right), 0.0\right\}
$$

and,

$$
f_{\text {rotation }}=\left(1+c_{r 1}\right) \frac{2 r^{*}}{1+r^{*}}\left[1-c_{r 3} \tan ^{-1}\left(c_{r 2} \tilde{r}\right)\right]-c_{r 1}
$$

and, $c_{r 1}(=1), \quad c_{r 2} \quad(=2)$ and $c_{r 3}(=1)$ were empirical constants[31,33]. Terms $r^{*}$ and $\tilde{r}$ were given as:

$$
\begin{gathered}
\tilde{r}=2 \Omega_{i j} S_{j k}\left[\frac{D S_{i j}}{D t}+\left(\varepsilon_{i m n} S_{j n}+\varepsilon_{j m n} S_{i n}\right) \Omega_{m}^{r o t}\right] \frac{1}{\Omega D^{3}} \\
r^{*}=\frac{S}{\Omega} ; \quad \Omega_{i j}=\frac{1}{2}\left(\left(\frac{\partial U_{i}}{\partial x_{j}}-\frac{\partial U_{j}}{\partial x_{i}}\right)+2 \varepsilon_{m i i} \Omega_{m}^{r o t}\right)
\end{gathered}
$$



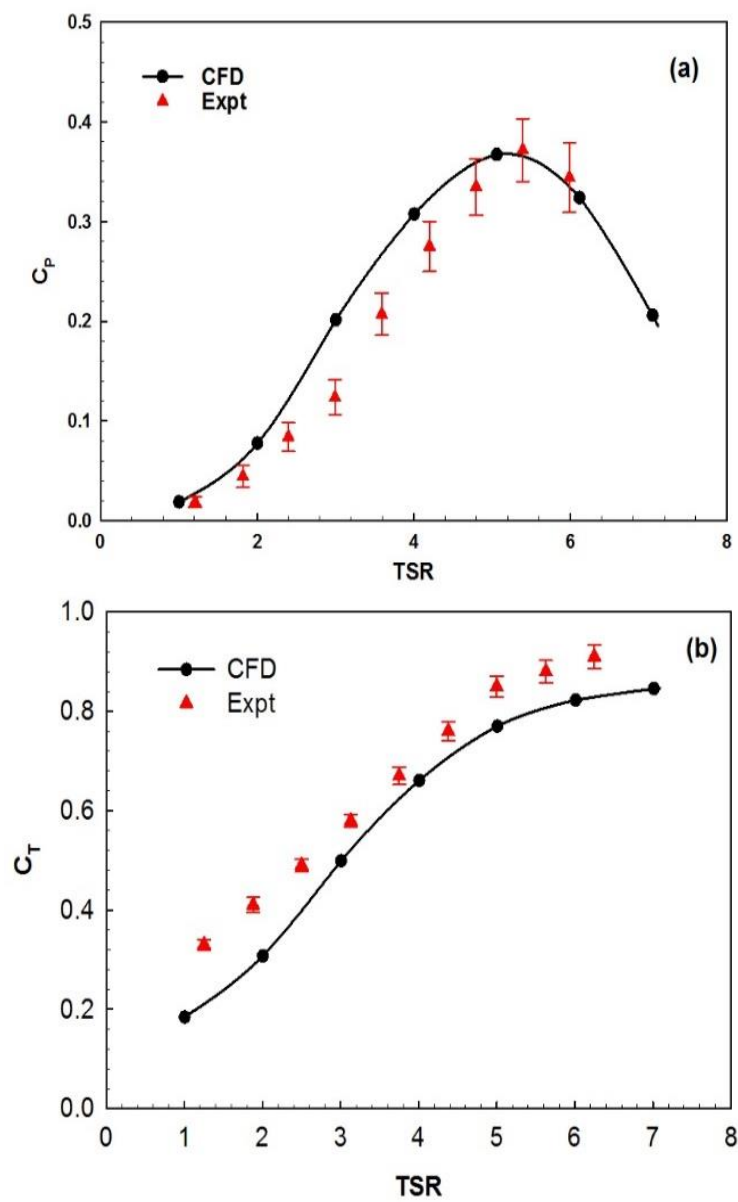

Figure 3: Comparison of (a) power coefficient $\left(C_{p}\right)$, and, (b) thrust coefficient $\left(C_{T}\right)$ obtained from CFD simulations with $\kappa-\omega$ SST turbulence model with curvature correction $(\mathrm{CC})$ with experimental data

where $S_{i j}$ was the strain rate tensor, $\Omega_{i j}$ was the rotation tensor, $\Omega_{m}^{r o t}$ was the rate of rotation of the system, w was the turbulent eddy frequency, and variable $D$ was defined as:

$$
D^{2}=\max \left(S^{2}, 0.09 \omega^{2}\right) ; \quad S^{2}=2 S_{i j} S_{i j} \text { and } \Omega^{2}=2 \Omega_{i j} \Omega_{i j}
$$

\section{Verification and Validation}

The $\kappa-\omega$ SST turbulence model with curvature correction (CC) model was validated for its ability to predict the performance of the turbine by comparing it with existing experimental data for $0^{\circ}$ yaw based on turbine performance calculation with the experimental data available in our laboratory [26]. Figure 3.a shows the performance curve of the turbine with freestream inlet velocity of $0.73 \mathrm{~m} / \mathrm{s}$ over a range of TSR values. From Figure 3.a it can be observed that the $\kappa-\omega$ SST-CC model was more accurate in predicting the performance at higher $T S R$ values. At the peak where the maximum value of $C_{\mathrm{p}}$ was observed, both computational and experimental values were comparable. After reaching the peak performance at $T S R=5$, the turbulence model predicts power coefficient values similar to experimental results, making it suitable for performance predictions at higher TSR. The
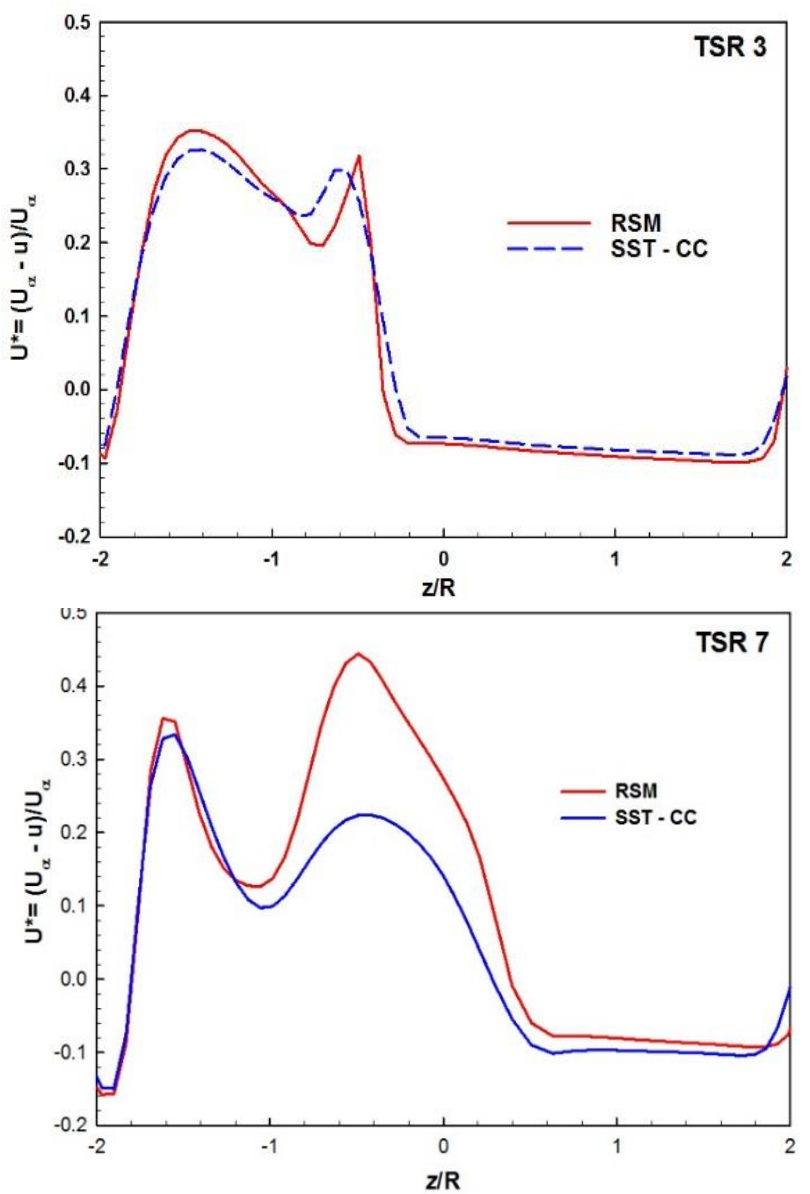

Figure 4: Comparison of wake deficit behind a turbine with $15^{\circ}$ yaw for TSR values of 3 and 7. Simulations performed with the $\kappa-\omega$ SST-CC model and a Reynolds Stress Model available in CFX. .

results obtained through computational methods were within the error factor of the experimental results obtained except between TSR of 2.5 to 4 . Figure $3 \mathrm{~b}$ shows the coefficient of thrust $\left(C_{\mathrm{T}}\right)$ curve of the turbine with a free stream inlet velocity of $0.73 \mathrm{~m} / \mathrm{s}$ over a range of $T S R$ values. From the plot, it can be observed that the computational model follows the same pattern as the experimental results with the error between them comparatively increasing from the TSR of max $\mathrm{C}_{\mathrm{P}}$ towards lower and higher TSRs. This variation in experimental and computational observations can be due to the presence of the enclosure and nacelle which cause variation in the drag force experienced. The difference between experimental and computational (CFD) results was lowest between $T S R$ of $4-5$ but increases as the rotational speed moves away for this TSR since it was close to design $T S R$ of the blade. The increase in thrust coefficient with increasing TSR was due to the higher drag force experience by the turbine with increasing rotational speed.

One deficiency of eddy viscosity formulations is the overly rapid vortex decay which leads to the incorrect prediction of the recovery rate of the wake [31]. The $\kappa-\omega$ SST-CC model was thus benchmarked with a Reynolds Stress Model developed by Speziale-Sarkar-Gatski (RSM-SSG) 
[34]. The RSM-SSG model was developed for aeronautical problems and directly solves for Reynolds Stresses without the eddy viscosity assumptions. Profiles of velocity deficit in the turbine wake for TSR values of 3 and 7 and are compared in Figure 4. It was observed that the wake velocity deficit [defined as $\mathrm{U}^{*}=\left(\mathrm{U}_{\infty}-\mathrm{u}\right) / \mathrm{U}_{\infty}$ ] profile is almost identical to $T S R=3$ case (see Fig.4, TSR=3 case). However, as the $T S R$ increased to 7 , the deviation between the predictions from the two models increased with the $\kappa-\omega$ SST-CC model showing a faster wake recovery when compared to the RSM-SSG case (see Fig. 4, TSR=7 case). The computational domain was thus chosen such that the wake of the yawed turbine was simulated up to a downstream location of $x=10 R$.

\section{RESULTS}

\section{A. Turbine Performance under Yaw}

The operation of a tidal turbine under yaw was quantified by studying variations in its performance by taking the zerodegree yaw case as a benchmark. Coefficients of power and thrust were studied to account for the power reduction caused due to the angular inflow of water. The skew in the wake propagation and recovery was understood by studying the velocity profiles and center of wake for yaw angles of $5^{\circ}, 10^{\circ}$ and $15^{\circ}$ at a rotational speed of $150 \mathrm{rpm}, 250 \mathrm{rpm}(T S R=5 \rightarrow$
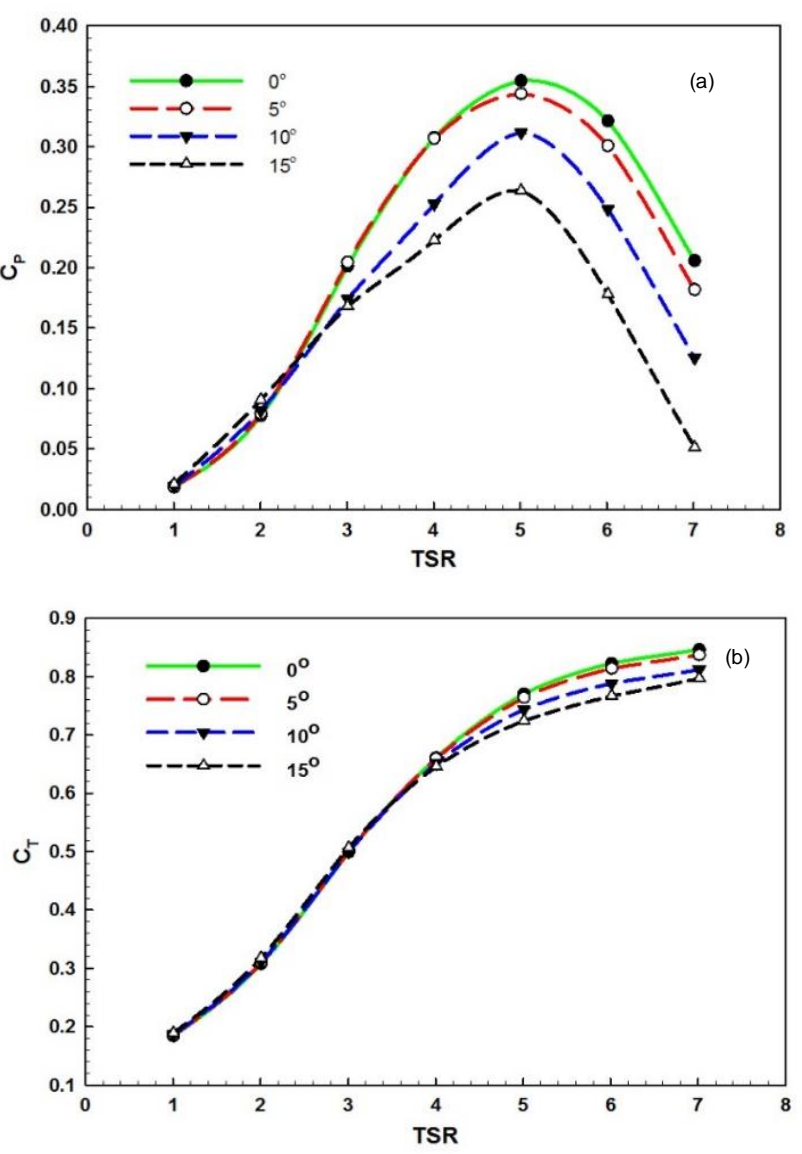

Figure 5: Effect of yaw angle on: (a) power coefficient $\left(C_{p}\right)$, and, (b) thrust coefficient $\left(C_{T}\right)$ of a horizontal axis tidal turbine. All simulations were run at uniform inlet velocity $U_{\infty}=0.73 \mathrm{~m} / \mathrm{s}$. maximum $C_{p}$ ) and $350 \mathrm{rpm}$ up to a downstream distance of $10 R$. Figure 5 a represents the performance curve of the turbine at different yaw angles. For each yaw angle, the rotational speed of the turbine was varied between, 50 to $350 \mathrm{rpm}$ (TSR 1-7). As reported by previous studies [9, 22], significant power reduction was observed as the yaw angle increased from $0^{\circ}$ to $15^{\circ}$. However, peak performance was observed at a TSR equal to 5 for all yaw angles. For the $5^{\circ}$ yaw case, there was no significant change observed in the turbine performance at lower values of $T S R(<4)$. However, the maximum output power was reduced by $4 \%$, and the turbine experiences small power reduction for higher $\operatorname{TSR}(\geq 5)$ when compared to $0^{\circ}$ yaw case. It was also observed that the effect of yaw angle on performance was not significant at lower values of $\operatorname{TSR}(<3)$; the change in performance $\left(\mathrm{C}_{\mathrm{P}}\right)$ for increasing yaw angle can be noticed to be less than $5 \%$ at all yaw angles. However, beyond a TSR value of 3 , a significant change can be noticed. A maximum power reduction close to $26 \%$ at peak output $(T S R=5)$ was observed at $15^{\circ}$ yaw. Observed power reductions varied from $16.5 \%$ at $T S R$ of 3 to about $75 \%$ for a TSR of 7 for the $15^{\circ}$ yaw case. A quadratic function was fitted to the peak $C_{p}$, max data $(T S R=5)$ and the turbine performance was related to the yaw angle $(\gamma)$ as:

$$
C_{p, \max }=0.3547-0.0002 \gamma-0.0004 \gamma^{2}
$$

Figure $5 \mathrm{~b}$ presents a comparison of thrust coefficient for
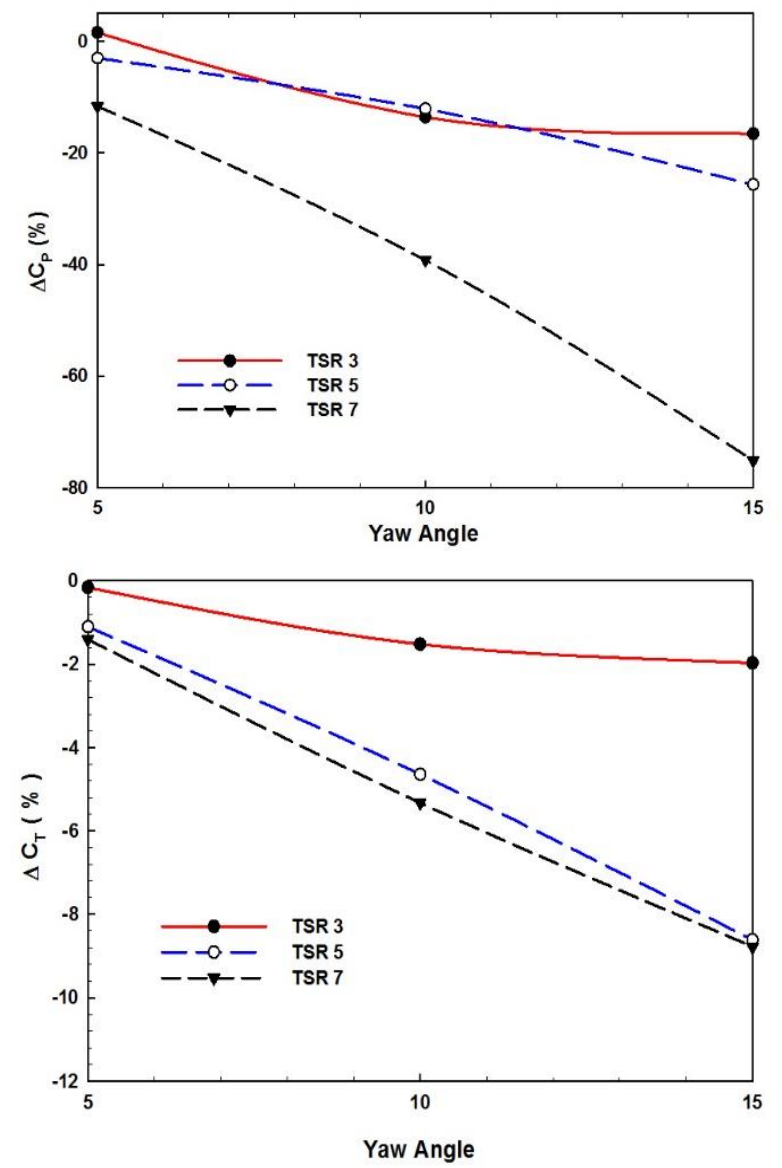

Figure 6: Percentage drop in (a) power coefficient $\left(C_{p}\right)$, and, (b) thrust coefficient $\left(C_{T}\right)$ of a horizontal axis tidal turbine due to yaw. 
different yaw angles when plotted against varying TSR. It was observed that at lower TSR $(<4)$, the thrust experienced by the turbine was not affected by the yaw angle. However, with an increase in turbine rpm, i.e., beyond TSR values of 4 , the thrust force experienced by the turbine decreases with an increase in yaw angle, with a maximum reduction of $8 \%$ at $T S R$ of 7 for $15^{\circ}$ yaw. Figure 6 a-b plots the power deficit and reduction in thrust experienced by the turbine with an increase in yaw angle. The deficit was evaluated with respect to the case of $0^{\circ}$ yaw. From the power deficit graph (fig.6a), it was observed that the rate of power reduction was strongly related to the yaw angle at high values of TSR (=7). At high TSR values, the blockage offered to the flow by the turbine can be felt upstream leading to larger deficits in the wake region and a corresponding reduction in power. The drop in the thrust coefficient is however much less with an increase of yaw (maximum difference of 6-8\%) and is not a string function of the blade TSR. The reduction in power and/or thrust is much higher than what would be expected if yaw angle effects were only assumed to be a function of the decrease of the projected swept area of the turbine rotor $\left(\cos \left(15^{\circ}\right)=0.965\right.$ which implies a $3.5 \%$ reduction in power).

\section{B. Wake Propagation}

\section{Stream-wise velocity profiles}

Figure 7 show contours of normalized stream-wise velocity $\left(U / U_{\infty}\right)$ plotted on horizontal $(X-Z)$ plane at $Y=0$ (hub height) for different yaw angles $\left(0^{\circ}, 5^{\circ}, 10^{\circ}\right.$ and $\left.15^{\circ}\right)$ with a uniform inlet flow of $U_{\infty}=0.73 \mathrm{~m} / \mathrm{s}$ and varying rotational speeds (TSR $=3,5,7$ respectively). $X$ and $Z$ axes were normalized with the turbine radius $(R)$. The increase in yaw angle resulted in an increase in skewness in the wake and a decrease in the wake recovery distance. Wake in the case of a horizontal axis turbine is divided into two regions; near wake (slow moving fluid region close to the rotor) and far wake (the region beyond the near wake). The region where large swirling eddies that were generated due to turbine rotor dissipates is called near wake region and is usually within 8$10 R$ downstream of the rotor [35]. The computational domain extends up to $10 R$ downstream from the turbine to allow characterization of the entire near-wake region. It was observed that as the wake propagates downstream, it deviates from the direction of yaw and this angle of deviation increases with an increase in the yaw angle. Upstream of the turbine ($1 \leq x / R \leq 0)$, the incoming flow is influenced by the rotor plane, and the level of interaction is dependent on the yaw angle and TSR. It is also observed that the width of the wake reduces as the wake propagates downstream; the width of the wake region decreases with an increase of the yaw angle. At the higher yaw angles, the increased wake deflection is likely to cause a more intense interaction between the freestream and the wake, accelerating the rate of momentum diffusion and quickening wake recovery. Similar observations were reported for a wind turbine by Lolland [13], in his study based on wind turbines under yaw where the wake was noticed to meander around the center line and deviate away from the ground as it travels downstream. Maximum wake recovery was noticed for $15^{\circ}$ yaw angle of the turbine at $9 \leq x / R \leq 10$. Notably, due to

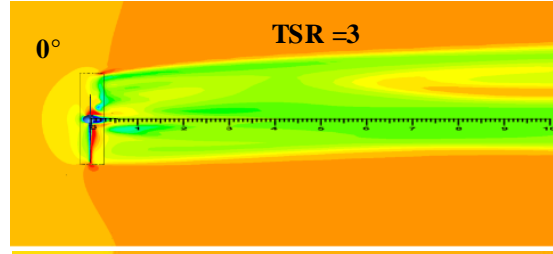

$5^{\circ}$
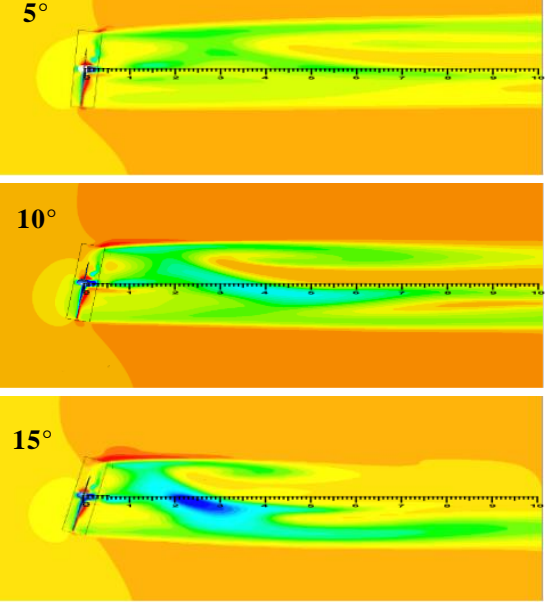

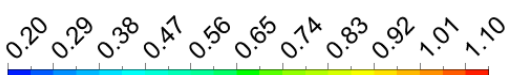

Figure 7: Stream-wise (horizontal) Velocity contours (Top View) - for various yaw angles at uniform inlet flow of $U_{\infty}=0.73 \mathrm{~m} / \mathrm{s}$
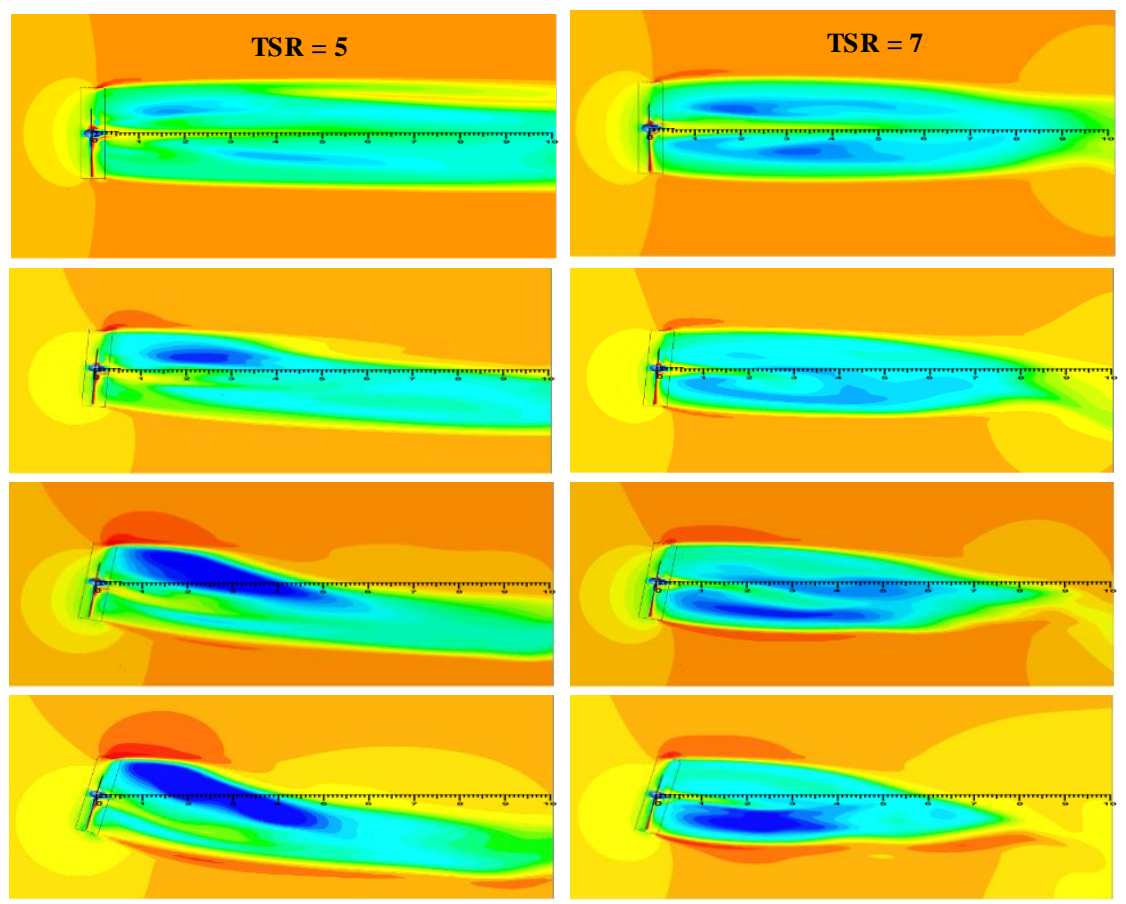

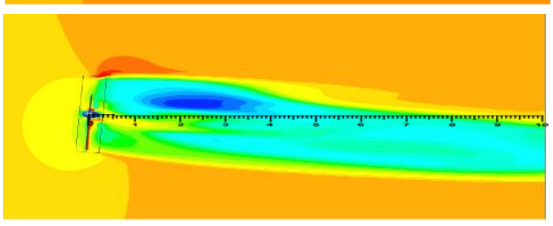

$\mathrm{U} / \mathrm{U}_{\infty}$

and $T S R=3,5,7$ respectively.

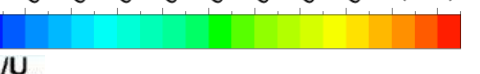


stream-tube expansion effects, the wake initially deviates in a direction opposite to turbine yaw (i.e., positive side of $Z$ axis); the momentum difference between the wake and the freestream (in the upper bypass region) causes a shift of the wake towards the direction of yaw. This shift is more gradual as the yaw angle increases with an observed reduction of wake width closer to this shift.

In addition, the extent of upstream influence increases with TSR and, is likely to be correlated to the increased blockage associated with TSR. At the higher TSRs analyzed (5 and 7), the region of upstream influence was observed to increases with yaw. Such a trend, however, was not noticeable at TSR of 3. Flow around the turbine can be observed to accelerate due to the blockage effects in the domain. One can also observe that the amount of deflection in the wake for a given yaw angle is largest at the maximum $C_{\mathrm{p}}$ point $(T S R=5)$; however, the wake recovery was observed to be faster up to $T S R=7$. In addition, a delay is observed before the wake turns towards the direction of yaw from the direction of flow; this distance is $\sim 2 R$ and is independent of the yaw angle. The delay may be attributed to the resistance offered by a faster moving bypass region to the deflected wake as it tries to penetrate it. The velocity deficit created in the wake at a downstream location was observed to increase in magnitude with increasing yaw and $T S R$ between $0 \leq x / R \leq 6$. With increasing $T S R$, the velocity deficit can be observed to extend to larger downstream distances in the wake; however, as the flow propagates downstream, the flow velocity was recovered faster for the higher yaw angles. For the zero yaw case, the velocity recovery over $1 \leq x / R \leq 10$, was about $10 \%$ recovery of the free-stream velocity. However, for yaw angle of $15^{\circ}$, the velocity recovered was $42 \%$ more recovery when compared to the zero-yaw case.

\section{Wake Analysis}

Investigating the change in the flow patterns of water after encountering the turbine rotor provides an advantage to decide the optimum location of turbines in a tidal farm. Propagation of wake was governed by the (yaw) angle of the turbine rotor to the flow, rotational speed and flow velocity of water. This study was conducted to understand how the varying yaw angle and rotational speed would affect the wake generated at a uniform velocity of water inflow. Center of the wake was tracked to understand the deflection of the near wake downstream of the turbine.

Wake propagation was initially studied by plotting velocity contours at different downstream locations for the rotational speed of $250 \mathrm{rpm}(T S R=5)$. Figure 8 presents the contour plots of wake development at different downstream locations $(x / R=1,5$, and 10$)$ at yaw angles of $0^{\circ}, 5^{\circ}, 10^{\circ}$ and 15. The location of the turbine was considered as a reference point $(0,0,0)$ and all other downstream locations are defined based on this reference point. It was observed that as the yaw angle increases, the velocity deficit behind turbine rotor is higher at the downstream locations closer to the turbine $(x / R<$ $5)$; the (wake) deficit recovery increases as the yaw angle increases. The increase in velocity caused by the tip vortices was directly proportional to the yaw angle at downstream locations in close proximity to the turbine. At downstream locations closer to the turbine rotor, the tip vortex can be clearly seen to interact with the surrounding flow, and the rise in velocity due to unsteady tip vortices increases with increasing yaw angle. The velocity deficit inside the wake (blue region in color map) was higher at higher yaw angles at locations closer to the rotor plane $(x / R=1)$. The interaction between the upper bypass region (region sandwiched between the wake and the free surface) and the wake increased with an increase in yaw angle leading to faster wake recovery. A close look at fig. 8 indicates traces of meandering as the wake propagates downstream. It is better reflected by the distribution of mean velocity in the wake, shifting the velocity deficit center sideways (between the side walls) more strongly than the top and bottom. While meandering, the wake was observed to shift upward (towards the free surface) and towards the left-wall as it moves downstream from the turbine rotor. Similar to the observations are reported by Trujillo et al. [18] for a wind turbine wake. From the normalized velocity contours at different downstream locations, the wake was observed to meander towards the angle of yaw as the wake propagates downstream. The meandering was in tune with the distribution of mean velocity in the wake, shifting the velocity deficit in the wake at the center sideways more strongly than the top and bottom. This meandering experienced by the wake increases with increase in yaw angle and can be attributed to set of counter-rotating vortices formed by yaw of turbine deforming the wake. Furthermore, a deviation of the wake towards the free surface was also observed with a maximum

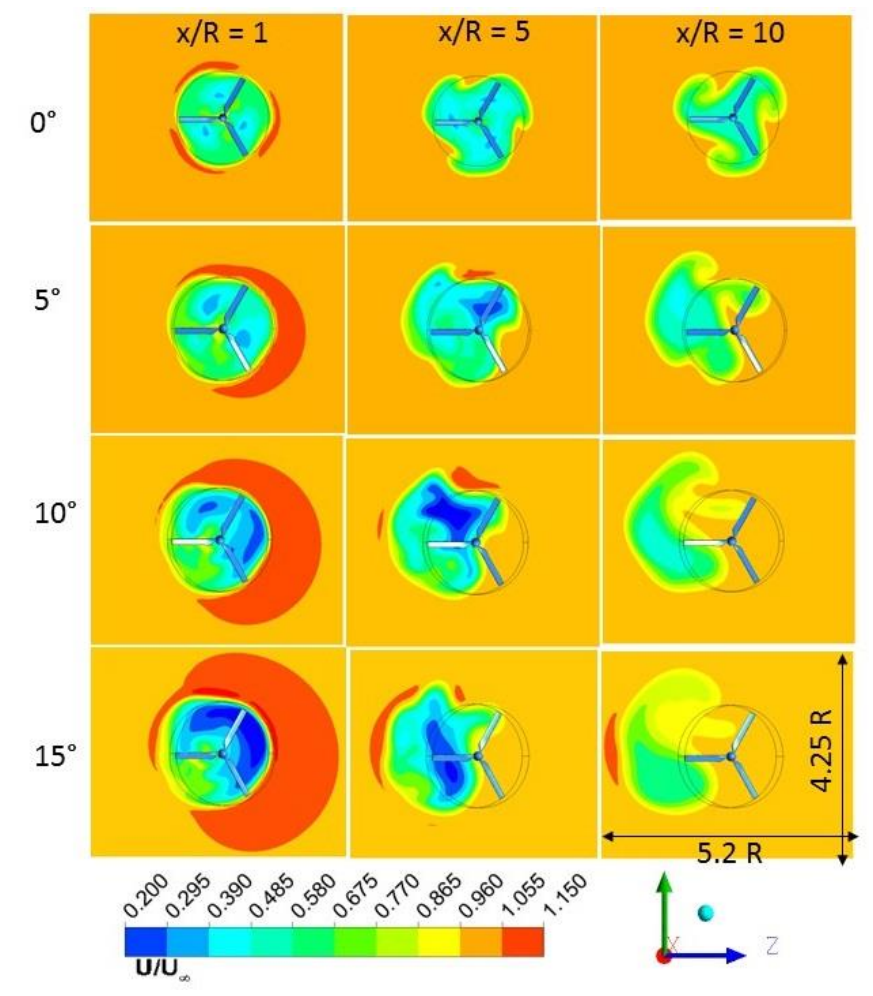

Figure 8: Normalized velocity contours showing wake deformation profiles at different downstream locations at $T S R=5$ (250 rpm) at different yaw angles. 
deviation of about $5^{\circ}$ for the case of $15^{\circ}$ yaw. These results were consistent with the results obtained for wind turbine experimentation conducted by Howland et al. [17] using an actuator disk model.

\section{Deflection of Center of Wake}

As stated earlier, with the yawed condition, the wake becomes asymmetric in span wise direction, making it difficult to characterize its deviation from the baseline case
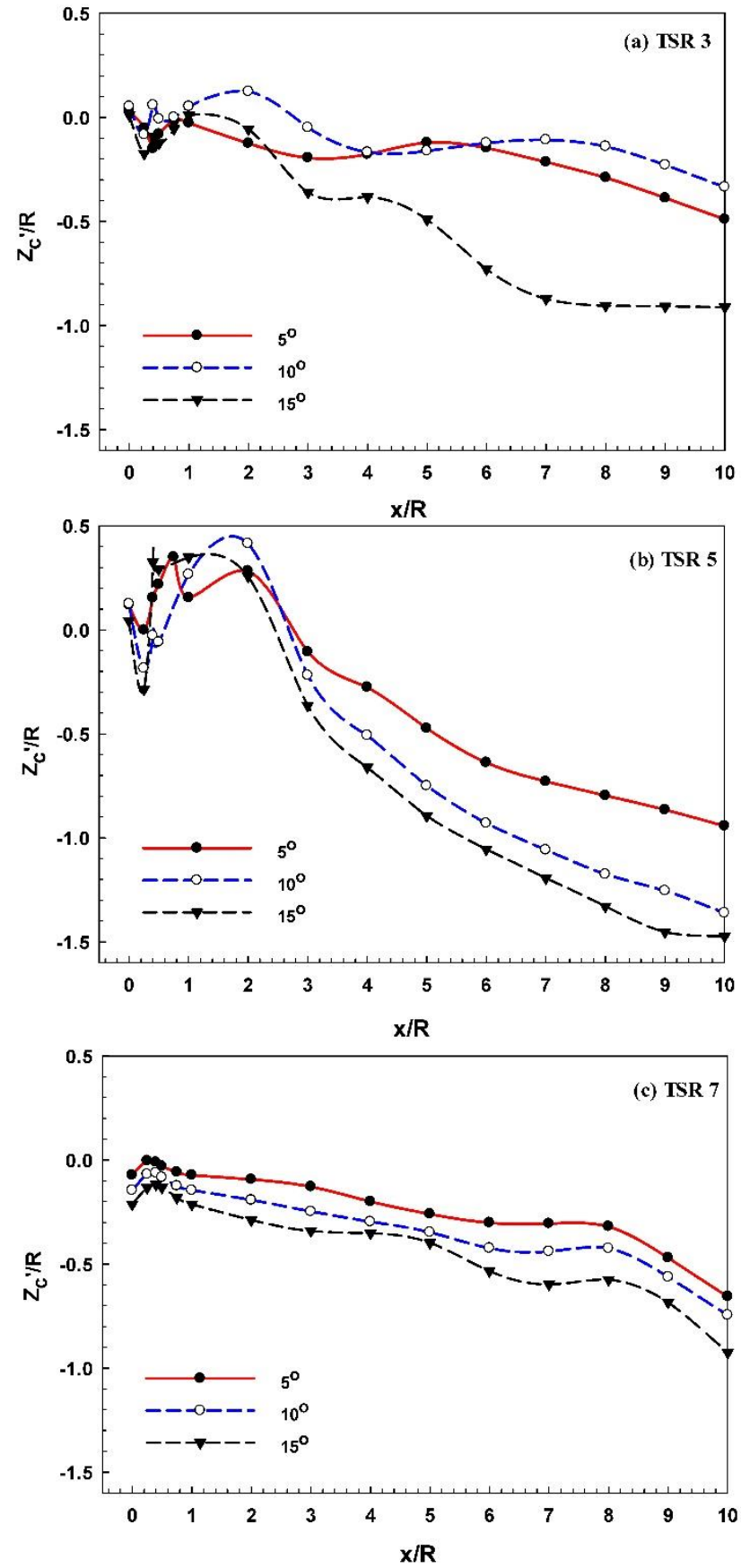

Figure 9: Comparison of wake deflection $\left(z_{c}{ }^{\prime}\right)$ for $\gamma=5^{\circ}, 10^{\circ}$ and $15^{\circ}$ tracked in $\mathrm{XZ}$ plane at hub height $(\mathrm{y} / \mathrm{D}=0)$ for (a) $T S R=3$, (b) $T S R=5$, and (c) $T S R=7$. $\left(0^{\circ}\right.$ yaw $)$ just based on its velocity profile. To better understand the deflection of the wake, it was necessary to track the center of the wake and compare it for various yaw angle cases. Several techniques are used to calculate the center of the wake, the more prominent methods include using a Gaussian fitting to the velocity deficit profile [36], velocity tracking [37], or estimating the center of mass of the velocity deficit (wake) region. According to Gaussian fitting, the velocity profile of the sections where the center was to be calculated was extracted, and the Gaussian center for it was regarded as the center of wake. In the velocity tracking method, the center of wake at a given cross-section was calculated as the mid-point between two locations with the local fluid velocity equal to $95 \%$ of free-stream velocity [37]. We choose a center of mass approach suggested by Howland et al. [17]. In this method, the coordinates of the center of wake were calculated at different downstream locations as:

$y_{c}(x)=\frac{\iint y \Delta U(x, y, z) d y d z}{\iint \Delta U(x, y, z) d y d z} \quad \& \quad z_{c}(x)=\frac{\iint z \Delta U(x, y, z) d y d z}{\iint \Delta U(x, y, z) d y d z}$

where $\Delta U(x, y, z)=U_{\infty}-\bar{u}(x, y, z) ; \quad \bar{u}$ is the timeaveraged velocity, and, $U_{\infty}$ was the free stream velocity. Integration was performed over the wake region- by assuming the wake width to be within $99 \%$ of the free-stream velocity. To obtain the wake center at different downstream locations (along the $x$ axis), we neglect $y$ dependency and use onedimensional integration in $z$ axis using,

$$
z_{c}^{\prime}(x)=\frac{\int z \Delta U(x, y=0, z) d z}{\int \Delta U(x, y=0, z) d z}
$$

Figure 9a-c shows the wake deflection coordinates $\left(z_{\mathrm{c}}{ }^{\prime}\right)$ at various downstream locations for different yaw angles at TSR values $3,5,7$ respectively. It was observed that wake deflection increases with increasing yaw angle. Initially $(x \leq$ $2 R$ ), the center of wake experiences a steeper deviation in a direction opposite to the yaw, this is confirmed through by cross verifying the wake center co-ordinates with the streamwise velocity contours of fig.7. Upon comparison, it was observed that initially, up to $x=2 R$, the center of wake experiences large variations. However, beyond $x>2 R$, the wake-center tends to meander slightly with a reduction in velocity deficit. The initial randomness is observed for all yaw angles with the deviations highest for $T S R=5$. As the wake travels downstream, the center of the wake stabilizes faster for larger values of yaw, suggesting that the velocity deficit recovery was more as discussed earlier in the previous section [13]. As the wake travels downstream (beyond $x / R=6$ ), the wake-deflection co-ordinates stabilize as the deficit in velocity is reduced, as can be observed for the $15^{\circ}$ yaw case at $T S R=3$. Wake deflection stabilization occurs due to the cushioning effect due to the tip vortices in the wake. As the turbine TSR is varied, it was observed that the yaw recovery of wake occurs at a faster rate as it travels downstream due to increased diffusion of freestream momentum into the wake. From the plots in fig 9, it can also be observed that, with increasing $T S R$, the influence of yaw decreases as the distance traveled by wake increases with the flow almost parallel to the free- 
stream at $x \sim 10 R$. However, based on the combination of TSR and yaw angle, a maximum offset of $z_{c}$ ' up to $1.5 R$ is observed. This information is useful for designing turbine layouts in a tidal farm.

\section{CONCLUSIONS}

The objective of the current study was to understand the performance variation and characterize the wake generated by a tidal turbine under yawed inflow conditions. A $\kappa-\omega S S T$ turbulence model with curvature correction was used. The results were benchmarked with experimental data for the case of a yaw angle of $0^{\circ}$. A rotating reference frame methodology was implemented to transform unsteady flow in the stationary frame to a steady flow in the rotating frame. A performance study was conducted by comparing the values of power coefficient $\left(\mathrm{C}_{\mathrm{P}}\right)$ and thrust coefficient $\left(\mathrm{C}_{\mathrm{T}}\right)$ under varying effects of $T S R$ and the yaw angle of the turbine. They were compared with experimental data [26] for zero degrees yaw turbine. Furthermore, wake propagation was studied with the help of normalized velocity, and velocity deficit contours and by tracking the center of the wake. The significant findings from this study can be summarized as follows:

a. Turbine performance was observed to decrease with increase in yaw angle. For individual yaw angle, the dependency of $\mathrm{C}_{\mathrm{P}}$ and $\mathrm{C}_{\mathrm{T}}$ on TSR remains the same, but when individual performance curve of different yaw angles was compared with each other, a power deficit was observed. A maximum deficit of close to $26 \%$ was found at $T S R$ value of 5 (that corresponds to the maximum $C_{P}$ ) between $0^{\circ}$ yaw to $15^{\circ}$ yaw. A maximum reduction of around $8 \%$ was experienced in thrust coefficient between $0^{\circ}$ and $15^{\circ}$ yaw angle at $T S R$ values of 7 .

b. Wake propagation was studied up to a downstream distance of $10 R$. From normalized velocity contours, it was observed that the wake recovery distance decreases with increasing yaw angle. This can prove to be useful in a farm setup where the distance between two downstream turbines can be reduced by placing a turbine yawed to the flow upstream; thereby increasing the farm turbine density which may subsequently lead to enhanced cumulative farm power output.

c. By studying wake propagation at different downstream locations $(1<x / R<10)$, it was concluded that the wake meanders as the downstream distance increases, with the center moving towards the direction of yaw. In addition, it was observed that the center of wake pitches up towards the free surface as it propagates downstream.

d. A center of wake analysis was used to quantify the wake recovery over a range of yaw angles. The results suggest that the wake tends to stabilize with increasing downstream distance for higher yaw angles which might be due to increased interaction of momentum from free stream velocity with the wake.

\section{ACKNOWLEDGEMENTS}

The authors would like to thank the U.S. National Science Foundation for financial support for this project (Award \# 1706358, CBET-Fluid Dynamics Program)

\section{REFERENCES}

[1] Electric Power Monthly Energy Information Administration, Vol. U.S. DoE, Washington D.C., 2016.

[2] D. Magagna and A. Uihlein, Ocean energy development in Europe: Current status and future perspectives, International Journal of Marine Energy, 11 (2015), 84-104.

[3] S. W. Funke, P. E. Farrell, and M. Piggott, Tidal turbine array optimisation using the adjoint approach, Renewable Energy, 63 (2014), 658-673.

[4] R. Bedard, M. Previsic, O. Siddiqui, G. Hagerman, and M. Robinson, Survey and characterization tidal in stream energy conversion (TISEC) devices, EPRI North American Tidal In Stream Power Feasibility Demonstration Project (2005),

[5] S. Gooch, J. Thomson, B. Polagye, and D. Meggitt, in OCEANS 2009, MTS/IEEE Biloxi-Marine Technology for Our Future: Global and Local Challenges, IEEE, 2009, p. 1-10.

[6] P. Galloway, Performance quantification of tidal turbines subjected to dynamic loading, Ph.D., University of Southampton, 2013.

[7] P. W. Galloway, L. E. Myers, and A. Bahaj, in Proceedings of the Renewable Energy Congress, Marine and Ocean Technology, 2011, p. 2246-2253.

[8] J. Norris and E. Droniou, in Proc. 7th European wave and tidal energy Conference, Porto, Portugal, 2007.

[9] P. W. Galloway, L. E. Myers, and A. S. Bahaj, Quantifying wave and yaw effects on a scale tidal stream turbine, Renewable Energy, 63 (2014), 297307.

[10] P.- $\AA$. Krogstad and M. S. Adaramola, Performance and near wake measurements of a model horizontal axis wind turbine, Wind Energy, 15 (2012), 743756.

[11] M. Adaramola and P.-А․ Krogstad, Experimental investigation of wake effects on wind turbine performance, Renewable Energy, 36 (2011), 20782086.

[12] D. Medici and J. Dahlberg, Potential improvement of wind turbine array efficiency by active wake control $(A W C)$, Proceedings of the European Wind Energy Conference and Exhibition (2003), 65-84. 
[13] K. M. Loland, Wind turbine in yawed operation, Norwegian University of Science and Technology, 2011.

[14] Results of sexbierum wind farm: double wake measurements, TNO-MT, Apeldoorn, Netherlands, 1992.

[15] D. Smith, in Proceedings of the 12th BWEA Wind Energy Conference. UK: Norwich, 1990, p. 53-56.

[16] D. Medici and P. Alfredsson, Measurements on a wind turbine wake: $3 D$ effects and bluff body vortex shedding, Wind Energy, 9 (2006), 219-236.

[17] M. F. Howland, J. Bossuyt, L. A. Martinez-Tossas, J. Meyers, and C. Meneveau, Wake Structure of Wind Turbines in Yaw under Uniform Inflow Conditions, Journal of Renewable and Sustainable Energy, 8 (2016), 043301.

[18] J. J. Trujillo, F. Bingöl, G. C. Larsen, J. Mann, and M. Kühn, Light detection and ranging measurements of wake dynamics. Part II: two-dimensional scanning, Wind Energy, 14 (2011), 61-75.

[19] W. M. J. Batten, A. S. Bahaj, A. F. Molland, and L. S. Blunden, in International Solar Energy Society UK Section Conference-C,, Vol. 85, 2006, p. 151.

[20] F. Maganga, G. Germain, J. King, G. Pinon, and E. Rivoalen, in 8th European Wave and Tidal Energy Conference, Uppsala, Sweden, 2009, p. 661-667.

[21] P. W. Galloway, L. E. Myers, and A. S. Bahaj, in World Renewable Energy Congress-Sweden; 8-13 May; 2011; Sweden, Linköping University Electronic Press, 2011, p. 2246-2253.

[22] W. Tian, J. H. VanZwieten, P. Pyakurel, and Y. Li, Influences of yaw angle and turbulence intensity on the performance of a $20 \mathrm{~kW}$ in-stream hydrokinetic turbine, Energy, 111 (2016), 104-116.

[23] W. Tian, J. H. VanZwieten, P. Pyakurel, and Y. Li, Influences of yaw angle and turbulence intensity on the performance of a $20 \mathrm{~kW}$ in-stream hydrokinetic turbine, Energy, 111 (2016), 104-116.

[24] S. Park, S. Park, and S. H. Rhee, Influence of blade deformation and yawed inflow on performance of a horizontal axis tidal stream turbine, Renewable Energy, 92 (2016), 321-332.

[25] C. Frost, C. E. Morris, A. Mason-Jones, D. M. O'Doherty, and T. O'Doherty, The effect of tidal flow directionality on tidal turbine performance characteristics, Renewable Energy, 78 (2015), 609620.

[26] N. Kolekar and A. Banerjee, Performance characterization and placement of a marine hydrokinetic turbine in a tidal channel under boundary proximity and blockage effects, Applied Energy, 148 (2015), 121-133.

[27] F. Menter, M. Kuntz, and R. Langtry, Ten years of industrial experience with the SST turbulence model, Turbulence, heat and mass transfer, 4 (2003), 625632.

[28] D. C. Wilcox, Turbulence modeling for CFD, DCW industries La Canada, CA, 1998.
[29] N. Kolekar and A. Banerjee, A coupled hydrostructural design optimization for hydrokinetic turbines, Journal of Renewable and Sustainable Energy, 5 (2013), 053146.

[30] T. P. Dhakal and D. K. Walters, A Three-Equation Variant of the SST $k-\omega$ Model Sensitized to Rotation and Curvature Effects, Journal of Fluids Engineering, 133 (2011), 111201-111201-111209.

[31] P. E. Smirnov and F. R. Menter, Sensitization of the SST Turbulence Model to Rotation and Curvature by Applying the Spalart-Shur Correction Term, Journal of Turbomachinery, 131 (2009), 041010-041010041018.

[32] P. R. Spalart and M. Shur, On the sensitization of turbulence models to rotation and curvature, Aerospace Science and Technology, 1 (1997), 297302.

[33] K. J. Elliott, E. Savory, R. J. Martinuzzi, and W. E. Lin, in 20th Annual Conference of the CFD Society of Canada, Alberta, 2012.

[34] C. G. Speziale, S. Sarkar, and T. B. Gatski, Modelling the pressure-strain correlation of turbulence: an invariant dynamical systems approach, Journal of Fluid Mechanics, 227 (1991), 245-272.

[35] L. Myers and A. Bahaj, in Proceedings of the 8th European Wave and Tidal Energy Conference, Uppsala, Sweden, 2009, p. 558-565.

[36] P. A. Fleming, P. M. Gebraad, S. Lee, J.-W. van Wingerden, K. Johnson, M. Churchfield, J. Michalakes, P. Spalart, and P. Moriarty, Evaluating techniques for redirecting turbine wakes using SOWFA, Renewable Energy, 70 (2014), 211-218.

[37] P. Parkin, R. Holm, and D. Medici, in Particle Image Velocimetry; Gottingen; 17 September 2001 through 19 September 2001, 2001, p. 155-162. 